\title{
The ZEUS Microvertex Detector
}

\author{
U. Koetz* \\ DESY, Hamburg Germany \\ E-mail: keetz@mail. desy.dé, \\ for the ZEUS Microvertex Group
}

\begin{abstract}
For the luminosity upgrade at the ep-collider HERA at DESY the ZEUS experiment has designed, constructed and recently installed a Silicon Microvertex Detector. The design of the vertex detector and the performance of prototypes are discussed, and first experience with the full detector using cosmic rays prior to installation are reported
\end{abstract}

\section{Introduction}

In parallel to the luminosity upgrade of the HERA $e^{ \pm} p$ collider at DESY the ZEUS experiment has improved its tracking system by installing the Si Microvertex Detector, MVD, and the Straw tube Tracker, STT. The barrel part of the MVD, the BMVD, will improve the overall precision of the central tracking system and allow the reconstruction of secondary vertices of long-lived heavy hadrons. In the forward (proton) direction the FMVD together with the STT will increase the acceptance and tracking capability for measurements in the high momentun transfer $Q^{2}$ region.

\section{Design and Construction}

The luminosity upgrade for HERA required that the last focussing magnets have to be installed inside the experiments. These magnets are the origin of very intense synchrotron radiation which cannot be intercepted anymore in front of the experiment and have, therefore, to be passed through the beampipe section of the experiments without hitting any obstacle. As a result the MVD is being built around a beampipe which is positioned off center from the central tracking with the particle beams passing through the beam pipe itself again off center. Fig. 1 shows the layout of the MVD: the three double-layered BMVD around the interaction point extending over $60 \mathrm{~cm}$ along the beam providing full acceptance for the long interaction region of about $20 \mathrm{~cm}$ and the forward part FMVD

\footnotetext{
${ }^{*}$ Speaker.
} 


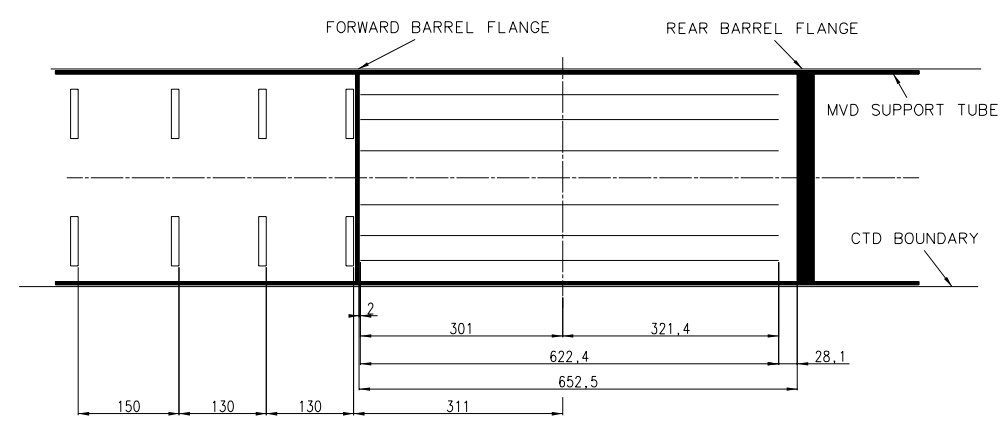

Figure 1: View of the MVD along the beam line
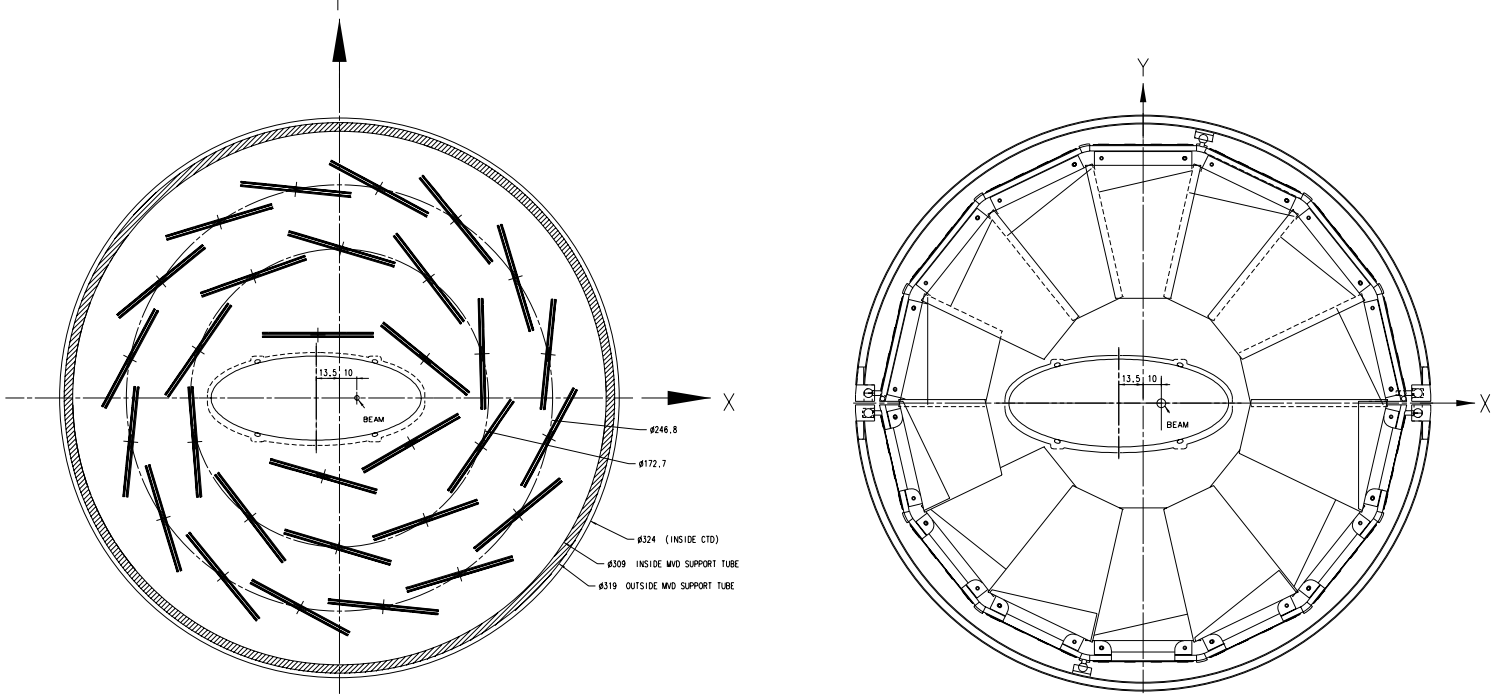

Figure 2: Cross section through the MVD: at the left the MVD, at the right the FMVD

consisting of four double-layered discs. The design of the BMVD and FMVD have to follow the elliptical, off center beampipe which results in an uncomplete inner most layer of the BMVD, Fig. 2. More detailed descriptions of layout, design and construction can be

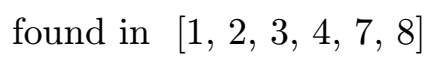

The design of the MVD aims for a point resolution of better than $20 \mu \mathrm{m}$ for each layer. Therefore sensors with a diode pitch of $20 \mu \mathrm{m}$ have been chosen. The single sided sensors are made from $320 \mu \mathrm{m}$ n-type silicon $(3-6 \mathrm{k} \Omega \mathrm{cm}$ with (111)-orientation) into which the $p^{+}$-diodes are implanted. Every 6th diode is capacitively coupled via a double layer of $\mathrm{SiO}_{2}$ and $\mathrm{Si}_{3} \mathrm{~N}_{4}$ to an Al-readout strip. Each $p^{+}$diode is connected via a polysilicon resistor of nominally $2 \mathrm{M} \Omega$ to a common voltage supply line. The sensitive area is surrounded by a triple guardring structure. The backside consists of a thick $n^{+}$diffusion. The BMVD has been constructed out of square shaped sensors of $64 \times 64 \mathrm{~mm}^{2}$ providing 512 readout strips per sensor whereas the FMVD consists out of trapezoidal sensors with 480 readout strips which are parallel to one of the edges. The precise position of a hit between the readout strips is determined by applying capacitive charge division. The Al-readout strips are connected by polyimid-microcables to the readout hybrid. The hybrid uses the readout 


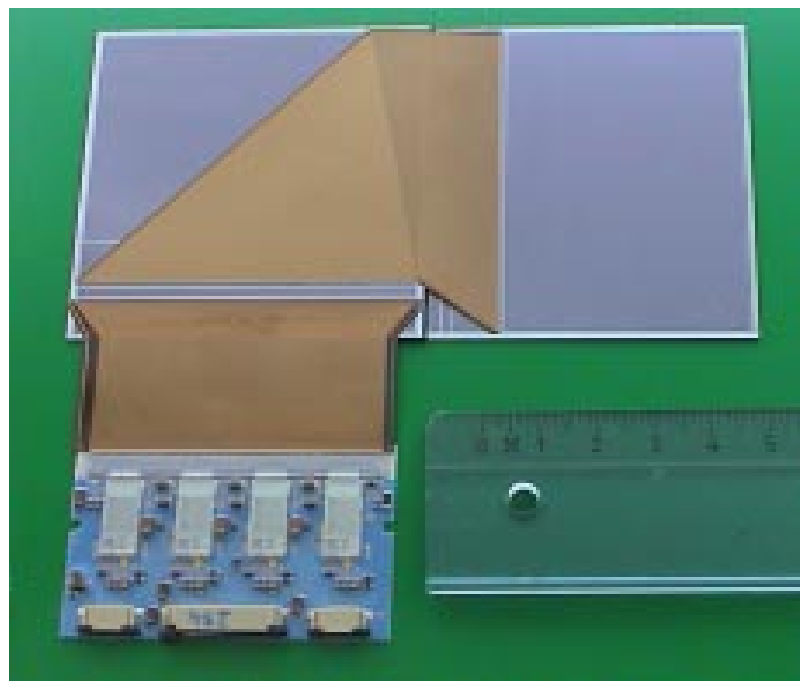

Figure 3: Photography of a halfmodule

chip Helix-128-3.0 which consists of 128 channels of preamplifiers and shapers, followed by

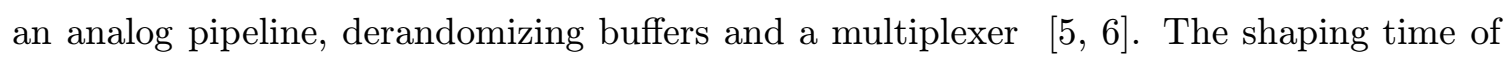
the input stage is about $60 \mathrm{~ns}$. The chip is characterized by a noise figure of $\operatorname{ENC}[e] \approx$ $400+40 * C[\mathrm{pF}]$. The readout electronics has to be positioned close to the sensors in order to mininimize the capacitive load to the preamplifier input. As a consequence the hybrid, its cabling and cooling add to the material in the active volume of the MVD which leads to multiple scattering.

Fig. 3 shows a photography of the basic structure of the BMVD, a halfmodule. Two sensors are glued together with a precision of $5 \mu \mathrm{m}$ with their diodes oriented perpendicular to each other. Two microcables connect the sensors to the readout hybrid. Two mirrorimaged halfmodules are mounted on top of each other to form the full module which provides space points without ambiguities. For the FMVD two sensors are mounted back to back to provide the space points. 150 full modules, arranged in 30 ladders, form the BMVD, in total 600 sensors. The FMVD consists of 112 sensors arranged in four discs. The amount of material for a particle traversing the BMVD at vertical incidence amounts to $3 \%$ of a radiation length.

\section{Measurements}

During the design and construction of the MVD an extensive test program has been performed. Besides the qualification controls of the sensors during the construction steps

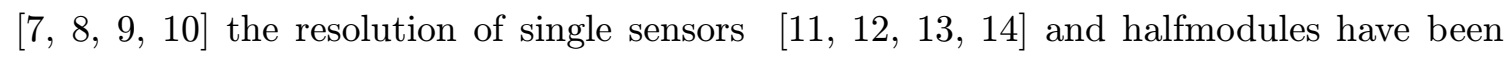
studied in detail in a testbeam at DESY providing electrons with momenta up to $6 \mathrm{GeV} / \mathrm{c}$. A precision telescope defined tracks with a precision of $3 \mu \mathrm{m}$. The measurements on single sensors resulted in an intrinsic resolution of $7 \mu \mathrm{m}$, corrected for multiple scattering, at vertical incidence. The signal/noise ratio $\mathrm{S} / \mathrm{N}$ was 22 . For halfmodules the $\mathrm{S} / \mathrm{N}$ decreased to 14 due to the about two times higher capacitive load at the input of the preamplifier. 


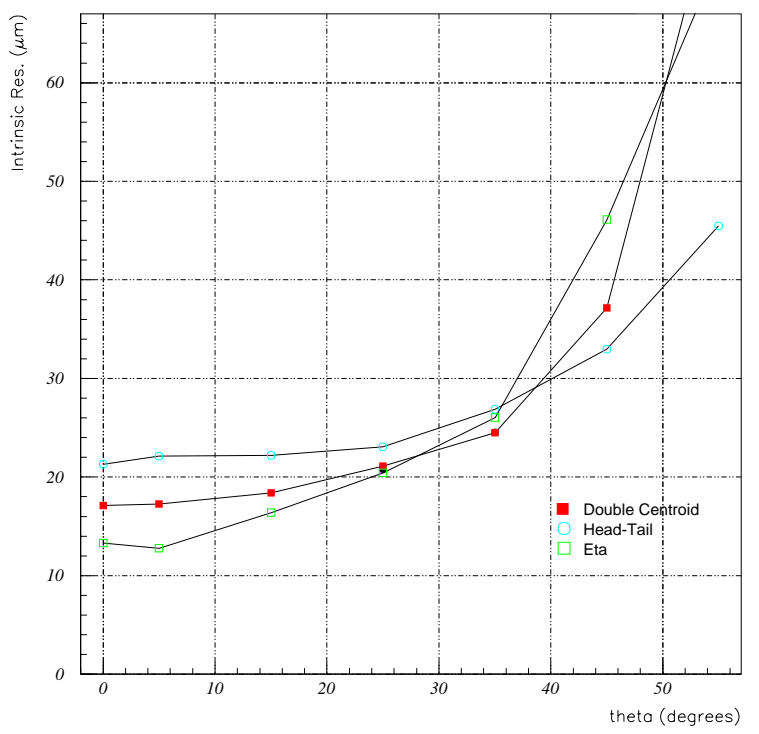

Figure 4: Resolution as function of angle of incidence using different reconstruction algorithms

Correspondingly the intrinsic resolution worsened to $13 \mu \mathrm{m}$ at vertical incidence. Fig.4 shows the angular dependence of the resolution for several reconstruction algorithms.

After completion and before installation into ZEUS a system test of the MVD was performed. For this purpose the MVD was tested thoroughly in its final configuration in a cosmic ray test setup. The momentum of the cosmic muons was not determined resulting in an average momentum of about $1.5 \mathrm{GeV} / \mathrm{c}$. Only $1 \%$ of the readout hybrids did not function properly. A S/N of 13 was measured. For resolution studies tracks were selected by requiring at least 6 hits with 2 good space points amongst them. The distribution of the residuals against the fitted track showed a rms of $70 \mu \mathrm{m}$. For the fit only the nominal positions of the sensors were taken into account, and no corrections were made for systematic shifts which have been documented at each step of the production procedure. Their effect on the resolution has been estimated to be about $50 \mu \mathrm{m}$. The low average momenta of the cosmic muons contributes as well via multiple scattering with an estimated $50 \mu \mathrm{m}$ to the resolution. These first measurements have shown that the alignment of the MVD is well understood and that the goal of a resolution of $20 \mu \mathrm{m}$ for one layer will be achieved. Final alignment studies will be done with high momentum tracks inside the ZEUS detector.

In spring 2001 the MVD has been installed into ZEUS. A very short cosmic run has proven that the MVD is fully operational as in the preceeding system test.

\section{References}

[1] A. Garfagnini, 'Nucl. Instrum. Meth. 435 
[2] C. Coldewey, 'Nucl. Instrum. Meth. $4 \overline{4} 7(2000)$ - 44

[3] C. Coldewey, iNucl. Instrum. Meth. 453 (2000) 149i

[4] E. Koffeman, 'Nucl. Instrum. Meth. 453 (2000)_89!

[5] M. Feuerstack-Raible, Helix 128S-2 User Manual, HD-ASIC-33-0697; http://wwwasic.ihep.uni-heidelberg.de/ feuersta/projects/ Helix/index.html

[6] M. Feuerstack-Raible, 'Nucl. Instrum. Meth. $\mathbf{4} \overline{4} \overline{7}(\overline{2} 0 \overline{0} 0)-\overline{3}$ !

[7] A. Garfagnini et al, Nucl. Instrum. Meth. 461

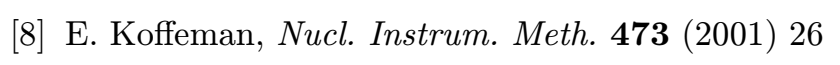

[9] D. Dannheim, DESY-THESIS-1999-027, 1999

[10] J. Martens, DESY-THESIS-1999-044, 1999

[11] R. Klanner, The ZEUS Microvertex Detector, Proceedings of the EPS-HEP99

[12] U. Koetz, 'Nucl. Instrum. Meth. 461

[13] I. Redondo Fernández, DESY-THESIS-2001-037, 2001

[14] M. Milite, DESY-THESIS-2001-050, 2001 